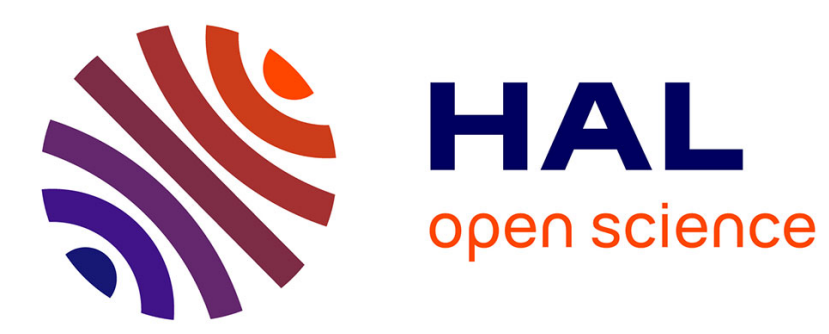

\title{
A rare case of deafferentation reveals an essential role of proprioception in bilateral coordination
}

\author{
Jacob Schaffer, F R Sarlegna, Robert Sainburg
}

\section{To cite this version:}

Jacob Schaffer, F R Sarlegna, Robert Sainburg. A rare case of deafferentation reveals an essential role of proprioception in bilateral coordination. Neuropsychologia, 2021, 160, pp.107969. 10.1016/j.neuropsychologia.2021.107969 . hal-03320962

\section{HAL Id: hal-03320962 https://hal.science/hal-03320962}

Submitted on 24 Aug 2021

HAL is a multi-disciplinary open access archive for the deposit and dissemination of scientific research documents, whether they are published or not. The documents may come from teaching and research institutions in France or abroad, or from public or private research centers.
L'archive ouverte pluridisciplinaire HAL, est destinée au dépôt et à la diffusion de documents scientifiques de niveau recherche, publiés ou non, émanant des établissements d'enseignement et de recherche français ou étrangers, des laboratoires publics ou privés. 
A rare case of deafferentation reveals an essential role of proprioception in bilateral coordination

\author{
Jacob E. Schaffer ${ }^{\mathrm{a}}$, Fabrice R. Sarlegna ${ }^{\mathrm{b}}$, Robert L. Sainburg ${ }^{\mathrm{a}, \mathrm{c}}$
}

a. The Pennsylvania State University, Department of Kinesiology

b. Aix Marseille Univ, CNRS, ISM, Marseille, France.

c. Penn State Milton S. Hershey College of Medicine, Department of Neurology

Address correspondence to:

Jacob E. Schaffer

27 Recreation Building

Penn State University

University Park, PA 16802

Email - jshafe11@gmail.com 


\section{Abstract}

Loss of proprioception has been shown to produce deficits in intralimb coordination and in the ability to stabilize limb posture in the absence of visual feedback. However, the role of proprioceptive signals in the feedforward and feedback control of interlimb coordination remains unclear. To address this issue, we examined bimanual coordination in a deafferented participant (DP) with large-fiber sensory neuropathy, which resulted in the loss of proprioception and touch in both arms, and in age-matched control participants. The task required participants to move a single virtual bar with both hands to a rectangular target with horizontal orientation. The participants received visual feedback of the virtual bar, but not of the hand positions along the bar-axis. Although the task required symmetrical movement between the arms, there were significant differences in the trajectories of the dominant and non-dominant hands in the deafferented participant, and thus more final errors and impaired coordination compared to controls. Deafferentation was also associated with an asymmetric deficit in stabilizing the hand at the end of motion, where the dominant arm showed more drift than the non-dominant arm. While the findings with DP may reflect a unique adaptation to deafferentation, they suggest that 1) Bilateral coordination depends on proprioceptive feedback, and 2) Postural stability at the end of motion can be specified through feedforward mechanisms, in the absence of proprioceptive feedback, but this process appears to be asymmetric, with better stability in the non-dominant arm.

Keywords: Proprioception - Large-fiber sensory neuropathy - Bimanual coordination - Motor lateralization - Handedness 


\section{Introduction}

The role of sensory information in motor control and coordination is reflected by the common use of terms such as sensorimotor control and sensorimotor cortices. The specific role of proprioception in motor control has been studied for over a century, and is emphasized by the extensive innervation of muscles with specialized sensors, including the muscle spindle and the Golgi tendon organ. Information from these sensors is transmitted to the central nervous system through the fastest neurons in the mammalian system such as type Ia, Ib, and II neurons, emphasizing the critical nature of proprioception to motor control (Boyd, 1965). Large Fiber Sensory Neuropathy is a rare condition that results in loss of proprioception and discriminative touch sensations. Typically, experimental studies have been performed on one patient, and revealed that the sensory loss produces deficits in everyday motor tasks such as walking (Lajoie et al., 1996) and unimanual motor skills such as object manipulation (Rothwell et al., 1982; Cuadra et al., 2019; Miall et al., 2019) and arm reaching (Blouin et al. 1993; Sarlegna et al., 2010; for a review, Sarlegna \& Sainburg, 2009). Patients deprived of somatosensory feedback also have impaired intralimb coordination, and impaired ability to stabilize the limb most notably but not only in the absence of visual feedback (Rothwell et al., 1982; Sainburg et al., 1993; Sainburg et al., 1995; Nougier et al., 1996; Sarlegna et al., 2006; Jayasinghe et al., 2020). A striking example of how deafferentation is associated with impaired coordination of multijoint arm movements was reported by Sainburg and colleagues $(1993,1995)$, who showed that deficits in interjoint coordination arises from a failure to take account of interaction torques between the moving limb segments. These authors suggested that proprioceptive feedback is required to prepare a motor plan that takes into account the mechanical effects arising from the inertial 
interactions between moving, linked limb segments, highlighting the role of proprioception in coordinating multiple elements (for reviews, Ghez \& Sainburg, 1995; Jayasinghe et al., 2021).

Bimanual movements add another layer of complexity to the system, as they require not only accurate interjoint coordination but also interlimb coordination. A few previous studies have examined the effect of deafferentation on bimanual movements, quantifying aspects of temporal coordination between the limbs. This approach was inspired by studies that have quantified "coupling" in bimanual movements in healthy people that appear to link certain aspects of each arm's movement (Kelso, Southard, \& Goodman, 1979b; Swinnen, Dounskaia, \& Duysens, 2002). Bozzachi et al. (2017) highlighted this coupling by reporting a strong correlation of the temporal aspects of bimanual movements. In fact, Helmuth and Ivry (1996) reported a "bimanual advantage" in healthy individuals, such that the variability in the timing of repetitive finger taps was decreased in bimanual tapping compared to unimanual (Helmuth \& Ivry, 1996). Drewing et al. (2004) also reported this timing advantage for bimanual tapping movements in deafferented participants, suggesting that such coupling can rely on feedforward processes which can updated without proprioceptive feedback, for instance with visual feedback. Spencer et al. (2005) showed intact temporal coupling for deafferented participants in a bimanual circle drawing task, but also highlighted the key role of proprioception to control spatial features of movement.

Seminal research by Diedrichsen and colleagues conducted in typical adults with intact proprioception, has shown how bimanual coordination requirements influence the control of spatiotemporal trajectory features (Diedrichsen, 2007; Diedrichsen \& Dowling, 2009). When participants had to reach simultaneously to two separate targets with each arm, the presentation of a perturbing force to one arm had no effect on the opposite arm. However, when participants 
moved a single cursor controlled with both arms, the opposite arm also corrected for the force perturbation, even though the limbs were not mechanically coupled. Mutha and Sainburg (2009), showed similar, but shorter latency effects, in response to discrete force pulses applied to one, but not the other arm. During a bimanual cooperative task, in which both hands work together to move a single cursor, proprioceptively-mediated reflexes were elicited in the opposite arm, but during a non-cooperative task of moving independent cursors, bimanual reflexes were not elicited. Schaffer and Sainburg (2020) recently corroborated these findings in a study that showed that perturbations applied to the dominant arm during a cooperative bimanual task elicited robust responses in the non-dominant arm. However, this study also found asymmetries in bimanual responses, as perturbations applied to the non-dominant arm did not elicit significant responses in the dominant arm. Overall, these studies suggest that coordination of cooperative bimanual movements relies on proprioceptively-mediated feedback responses. In addition, these studies suggest that cooperative bimanual tasks may recruit unique neural mechanisms that rely on task-dependent sensory feedback coming from both limbs.

The current study aimed to specifically examine how a lack of proprioception influences bimanual coordination. Considering the previous findings, along with previous work on deafferentation showing that proprioception is essential in intersegmental coordination (Sainburg et al., 1993, 1995), we hypothesized that lack of proprioception from each limb resulting from deafferentation would result in less coordinated, and more asymmetric movements between the limbs. during a bimanual task that requires shared control of object position and orientation. We examined how proprioception contributes to bimanual coordination of goal-directed movements by assessing the performance of a proprioceptively-deafferented patient in a cooperative bimanual task which we have recently characterized in older participants (Schaffer et al., 2020). 
In the present study, the deafferented participant performed bimanual movements in which both arms were used to move a shared object (bar) to a target area with a precise orientation. The performance of the deafferented participant was compared to that of an age-matched control group. 


\section{Methods}

\section{Participants}

We tested a deafferented participant, (DP; female; 70 years old; right-hand dominant), who has been living with large-fiber sensory neuropathy for 40 years following an acute postviral onset at age 31 , along with 5 healthy age-matched control participants (age $67 \pm 2.9 ; 2$ males, 3 females). Clinical tests of DP revealed a specific loss of large-diameter, myelinated afferents which resulted in a complete loss of touch, vibration, pressure, tendon reflexes, and sense of movement and position in the four limbs, the trunk being moderately affected (Cooke et al., 1985). Such loss is associated with deficits in everyday tasks, as detailed in numerous reports (Cole \& Paillard, 1995; Cuadra et al., 2019; Miall et al., 2019). We received written informed consent from all participants prior to the study. All study procedures were approved by the Pennsylvania State University’s Institutional Review Board.

\section{Experimental set-up}

Participants were seated at a 2-D virtual-reality workspace in which stimuli from a HDTV screen were reflected by a mirror, with the participants' arms under the mirror (Figure 1). Participants' arm movements were tracked using 6 DOF magnetic sensors (Ascension trakSTAR) placed on the hand and upper arm. All joints distal to the forearm were splinted. We digitized the location of the tip of the index finger, as well as multiple locations on the hand and upper arm, and used custom software to estimate the locations of the wrist, elbow, and shoulder joints, relative to these digitized landmarks. Vision of the participants' arms was occluded while position of the index finger was provided as a cursor on the screen. Participants' arms were 
supported on air sleds that reduced the effects of friction, and eliminated gravitational torques at the joints.

The experimental session consisted of 80 total bimanual movements. The task required participants to first "grab" a virtual bar $(20 \mathrm{~cm}$ across $)$ by moving cursors representing the position of each hand to each end of the bar. Next, participants moved the bar into the start position. After maintaining the bar in the start position for $100 \mathrm{~ms}$, participants were given an auditory start signal and the cursors disappeared, giving participants visual feedback of only the bar. The task required participants to move the bar with both hands toward a target trough that was $15 \mathrm{~cm}$ away from the start position. Points were given for accuracy, as long as the bar's maximum velocity reached at least $0.8 \mathrm{~m} / \mathrm{s}$. Maximum velocity feedback of the bar movement was displayed as a progress bar, with a target region, at the top of the screen, after each trial. Average movement time was 0.63 seconds for control participants, and 0.79 seconds for the deafferented participant.

Accurate performance in this task required precise interlimb coordination in both the $\mathrm{x}$ and y-axes (see Figure 1B). Participants had to use both hands together to move the shared bar the proper distance in the y-axis, and also control the orientation of the bar by ending with each hand at the same y-position. The ability to maintain a horizontal bar orientation reflected the coordination between the hands in the y-axis. In addition, as shown in Figure 1, movement along the long axis of the bar was redundant, allowing the fingertips to move outside of the bar in the redundant axis of the bar. However, to keep the bar in the target $\mathrm{x}$-position required coordination between the hands such that any movement in the x-direction of the bar in one hand had to be countered by an equal and opposite movement in the other hand so that the bar would not deviate in either direction. 

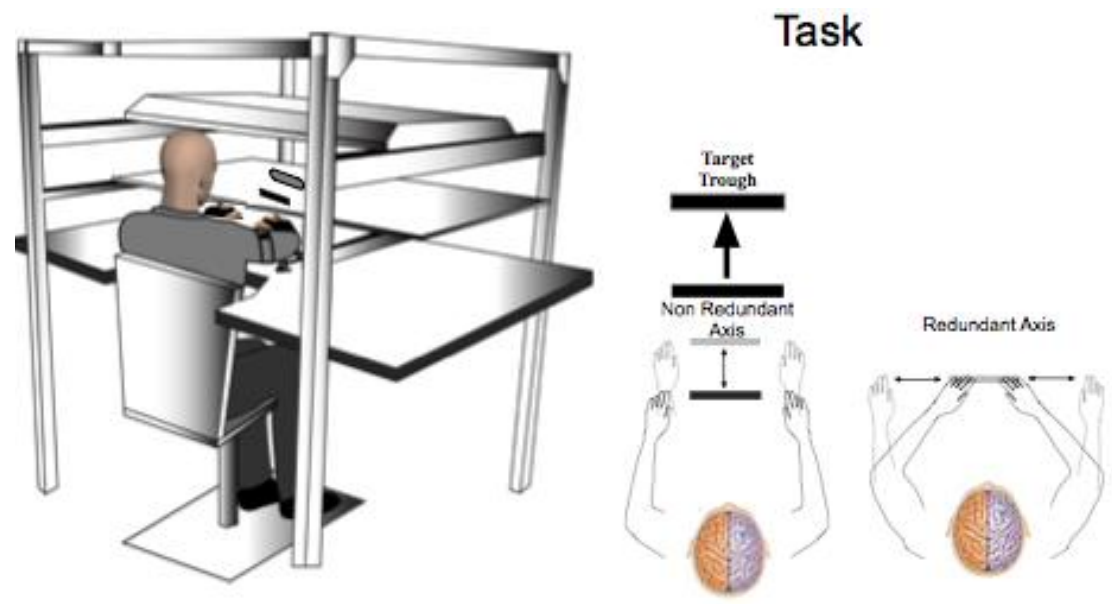

Figure 1. Experimental setup. The left panel shows a HDTV screen positioned above a mirror, creating a 2-D virtual reality workspace. The right panel shows how the participants could move the virtual bar toward the target trough.

\section{Kinematic Analysis}

We calculated arm segment positions and angles from digitized locations relative to the Ascension trakSTAR 6-DOF sensors. Data were collected from each sensor at $116 \mathrm{~Hz}$. We digitized multiple positions on the hand, wrist and upper arm. Using custom software, we calculated 10 degrees of freedom per arm but, because this task was restricted to the horizontal plane by air sled support and all joints distal to the forearm were splinted, we report only planar motion of the hand, as well as horizontal flexion/extension of the shoulder and elbow joint flexion/extension. All kinematic data were low-pass filtered at $8 \mathrm{~Hz}$ (3rd order, dual pass Butterworth) and differentiated to yield velocity and acceleration.

Main task performance measures included bar distance error, which measured the distance of the center of the bar from the center of the target trough, and bar orientation error which measured the angle of the bar with respect to the horizontal. Bar orientation reflects the ability of the participants to coordinate movements of both hands in the y-axis. The ability to coordinate movements along the axis of the bar of the left and right hands was also important for 
accuracy of the task. Success in this cooperative task required negative covariation between the hands to limit deviation of the bar along the redundant $\mathrm{x}$-axis. To quantify the relationship between the arms we plotted left hand vs. right hand movement along the redundant axis of the bar at peak velocity and at the end of movement and analyzed the correlation between the two hands, using orthogonal regression analysis. We also analyzed the trajectory of each hand path by calculating the hand path deviation from linearity. Deviation from linearity was assessed as the minor axis divided by the major axis of the hand path. The major axis was defined as the largest distance between any two points in the path, while the minor axis was defined as the largest distance, perpendicular to the major axis, between any two points in the path (Sainburg, 2002).

Drift of the hand between initial movement termination and the end of the trial was also calculated. Initial movement termination for each hand was defined as the first minimum $(<5 \%$ maximum tangential velocity) following peak tangential hand velocity. We calculated drift in two ways: 1) cumulative drift, which was calculated by adding the point to point distance travelled by the hand for each datapoint from the initial movement termination point to the end of the trial, and 2) average drift, which was calculated as the displacement of the hand from the initial stop to the end of the trial, using just those two datapoints. This was done in order to characterize whether the hands drifted away from the endpoint or oscillated around the endpoint.

\section{Statistical Analysis}

Data comparisons between the deafferented patient and controls of bimanual measures of task performance such as bar distance error and bar orientation were done using the Crawford method (Crawford et al., 2010). Such method was developed for comparison of a single- case's 
score to scores obtained in a modestly-size matched control sample, and also is robust against departures from normality. This method has been employed in similar studies with single casecontrol study designs (Cuadra et al., 2019; Lafargue et al., 2003; Jayasinghe et al., 2020).

We also analyzed differences in performance between the hands within control participants. Within-subject comparisons of single subject data using parametric tests are highly prone to Type 1 errors. In order to assess difference between the hands for the deafferented patient, we used the revised standardized difference test (RSDT; Crawford \& Garthwaite, 2005). This test implements classical methods to test for a difference between a single- case's scores in two conditions, which in this task is the difference between the hands, by comparing the difference against differences observed in the control sample. 


\section{Results}

All participants were instructed to move the bar into the target trough and maintain accurate final position throughout the duration of the trial. While control participants accurately moved the bar into the target trough and held the position with little drift, the deafferented patient was less accurate and the movements of her dominant and non-dominant arms appeared less symmetric. In addition, the deafferented participant showed substantial drift in final position associated with submovements following the primarily forward motion, and this drift was greater for the dominant arm compared to the non-dominant.

Figure 2 shows example movements for deafferented and control participants with lines representing stick figures of the arm. Below the example movements are the hand velocities shown for each hand. In the velocity profiles, we separated the main component of movement that acted to move the bar forward from the start position to the target trough (black line) from the subsequent, smaller submovements (gray line). A large, initial velocity peak was associated with the primary movement, as clearly shown for controls and the deafferented participant (figure 2, bottom panels). The greater submovements and their asymmetry in the deafferented participant compared to controls are reflected in the larger secondary velocity peaks of the deafferented participant, most notably for the dominant right arm. 


\section{Deafferented}

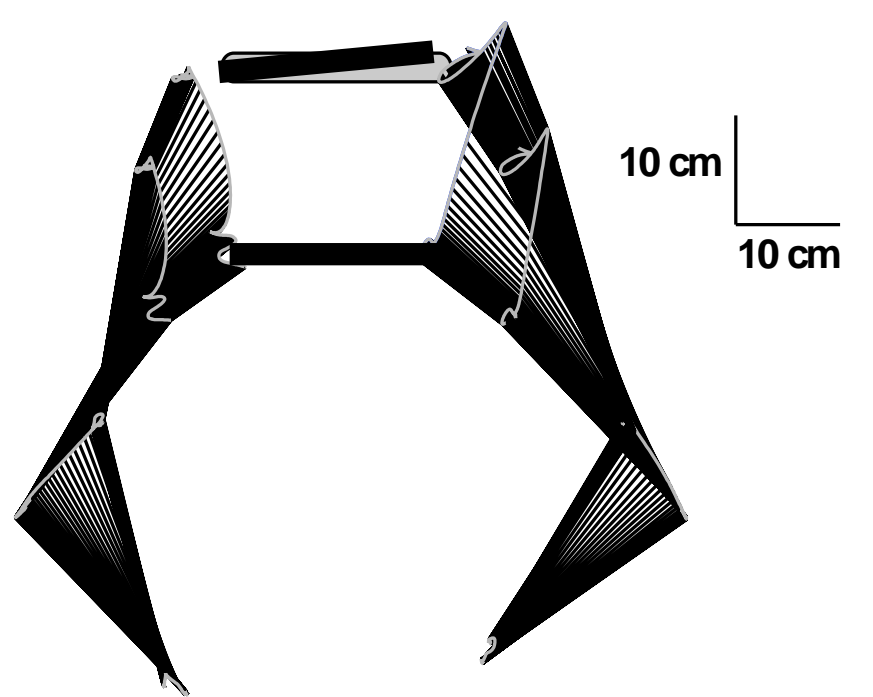

Left Hand Velocity

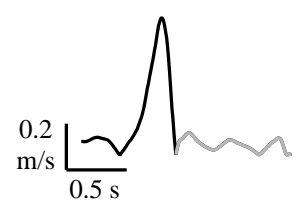

Right Hand Velocity

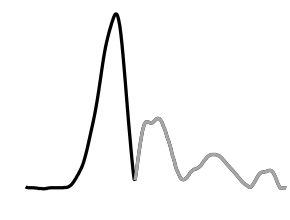

\section{Control}

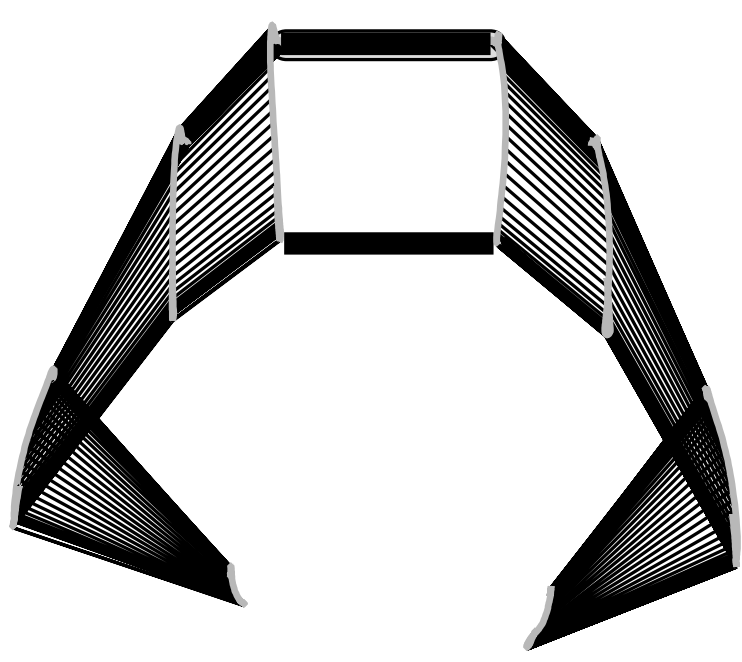

Left Hand Velocity

Right Hand Velocity

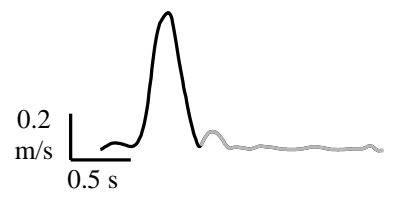

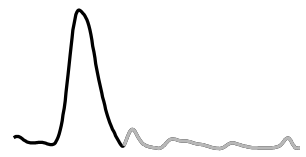

Figure 2. Example movements of the Deafferented patient and a Control participant. Example movements are shown (top view) with stick figures of the arms drawn between every two data points $(17.2 \mathrm{~ms})$, along with velocity profiles for each hand. The black part of the velocity profile reflects the main component of movement, from start to initial stop of movement. The gray part reflects the submovements or drift that occurred after initial movement stop.

Final errors are larger in the deafferented patient compared to controls

Figure 3 shows the main task performance measures including the final position error of the bar and the absolute value of orientation of the bar throughout the movement. Both measures were important for task success, as the goal was to move the bar the correct distance while also keeping it horizontally oriented to align with the target trough. Case-controls comparison with the Crawford method showed that the deafferented participant had significantly larger errors in final position of the bar at movement end compared to controls $(\mathrm{p}=.029)$. The deafferented participant also showed significantly increased bar orientation error at bar peak acceleration $(\mathrm{p}=$ 
$.00474)$, and peak velocity $(\mathrm{p}=.00642)$. The absolute orientation of the bar at movement end did not significantly differ from control participants $(\mathrm{p}=.129)$. It should be noted that there were no significant differences between the patient and controls in the magnitude of peak velocity $(\mathrm{p}=$ $.895)$ and peak acceleration $(\mathrm{p}=.331)$, nor was there any significant difference in the timing of peak velocity $(\mathrm{p}=.316)$ and peak acceleration $(\mathrm{p}=.588)$.
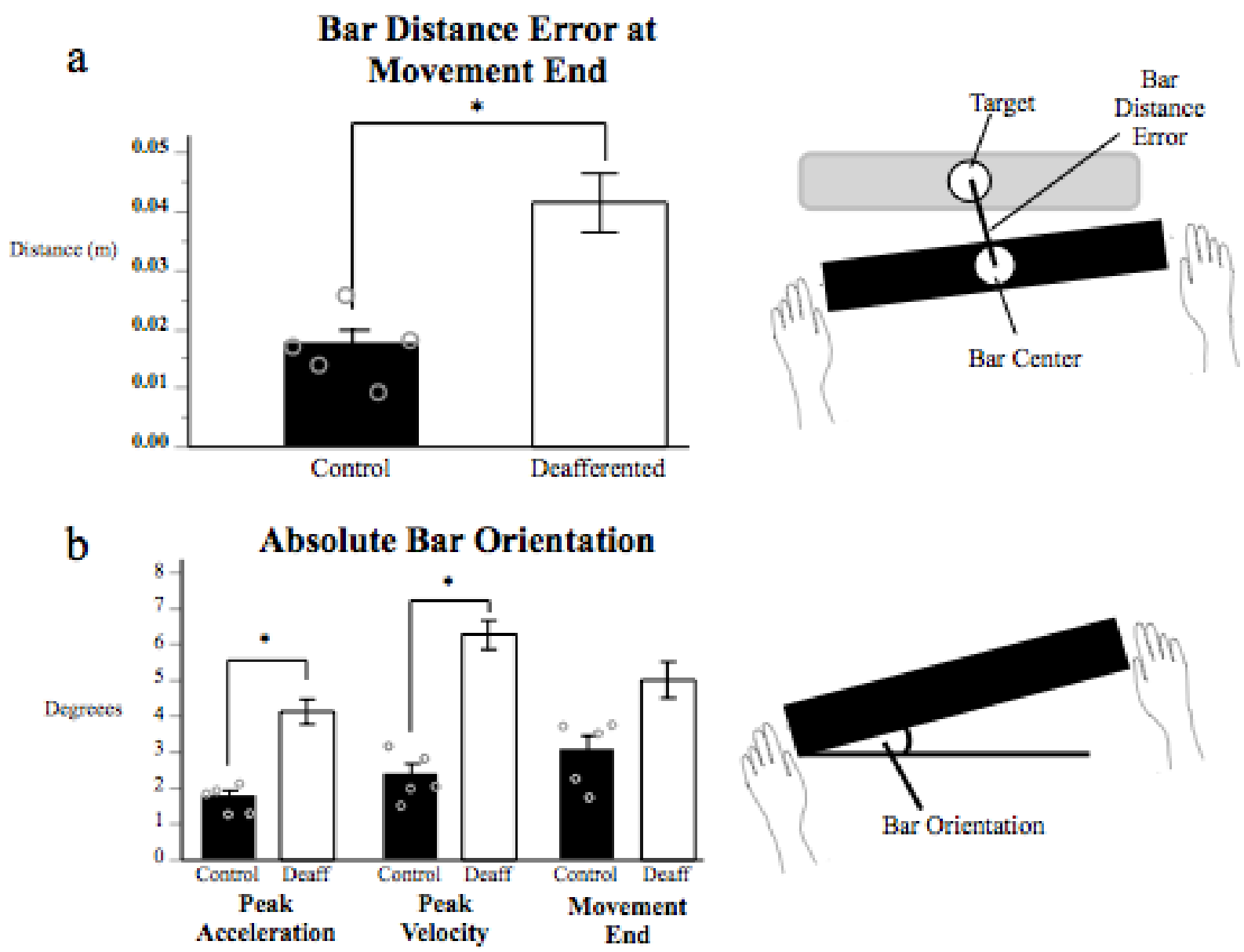

Figure 3. Task Performance. a) Mean distance error of the bar at the end of movement across participants for the Control group and across all trials for the Deafferented participant. b) Absolute value of the bar angle at various points in the movement. Error bars reflect standard error across participants for the Control group and across trials for the Deafferented participant. Gray open circles represent means of individual control participants. 
Figure 4 shows spatial measures for the movements of both hands. The control group showed significantly lower deviation from linearity, and lower directional deviations, in both hands than the deafferented participant. A paired t-test on controls' data revealed no significant differences between arms $(\mathrm{p}=.5443)$. In contrast, we found that the linearity difference between the hands for the deafferented participant was significantly larger than for the control group using the RSDT $(\mathrm{p}=.019)$. While a similar trend was seen in the absolute direction deviation at peak velocity, this asymmetry did not reach significance $(\mathrm{p}=.073)$. The asymmetry in hand trajectories was likely related to the increase in bar angle of the deafferented participant throughout the movement compared to controls. 


\section{Hand Trajectories}
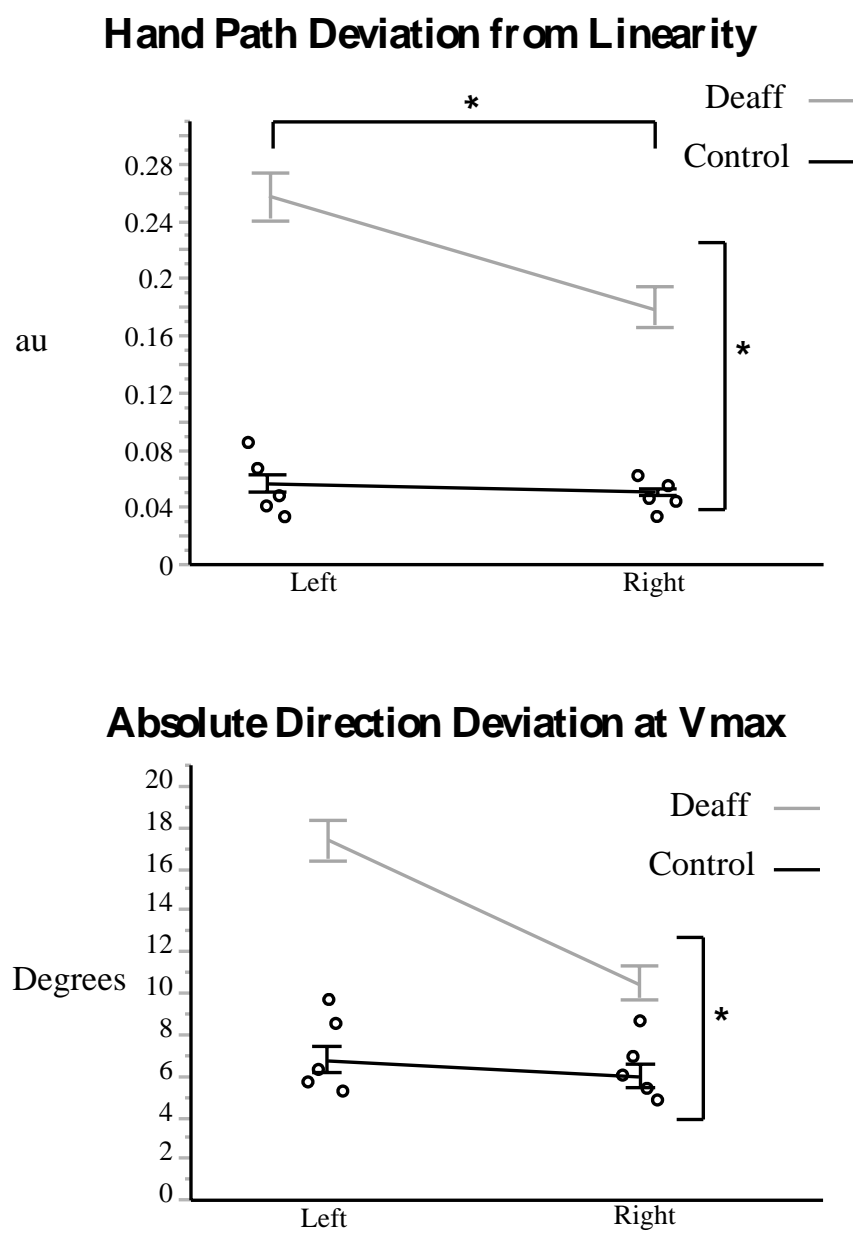

Figure 4. Kinematic analysis of hand trajectories for both arms. The top panel shows the mean values of hand path deviation from linearity and the bottom panel shows the absolute value of direction deviation at peak velocity for both arms. Error bars reflect standard error across all participants for the Control group and across trials for the Deafferented patient. Black open circles represent means of individual control participants.

Drift is larger in the deafferented patient compared to controls

Deafferentation was associated with pronounced differences in stabilizing the hand at the end of motion. The velocity profiles of Figure 2 show substantial submovements at the end of the initial motion, during which the deafferented participant's dominant right hand tended to drift away from the initial stop position more than the left hand. Figure 5 shows example movements 
that illustrate the typical pattern of movement for controls and deafferented participants after the initial stop of movement. The black portion of the hand paths in the example movements show the main component of the movement from start to the initial stop, while the positional drift, following this initial primary motion, is shown in gray. We calculated drift as cumulative distance travelled by the hands from the end of the primary movement until the end of the trial (cumulative drift) and also as the displacement from the position of the hand at the end of the primary movement to the position of the hand at the end of the trial (average drift) in order to characterize whether the hands drifted away from the endpoint or oscillated around the endpoint. Figure 5 shows both of these measures. Case-controls comparison showed that, compared to controls, the deafferented participant had significantly larger drift for the dominant, but not the non-dominant arm than controls. The paired t-tests on control data revealed no significant differences between arms for cumulative drift $(\mathrm{p}=.8187)$ and average drift $(\mathrm{p}=.6326)$. For cumulative drift, RSDT revealed that the differences between the hands for the deafferented participant were larger than for the control group $(\mathrm{p}=.018)$. Average drift also showed that the asymmetry in the deafferented participant was greater than that of the control group $(\mathrm{p}=.002)$. 

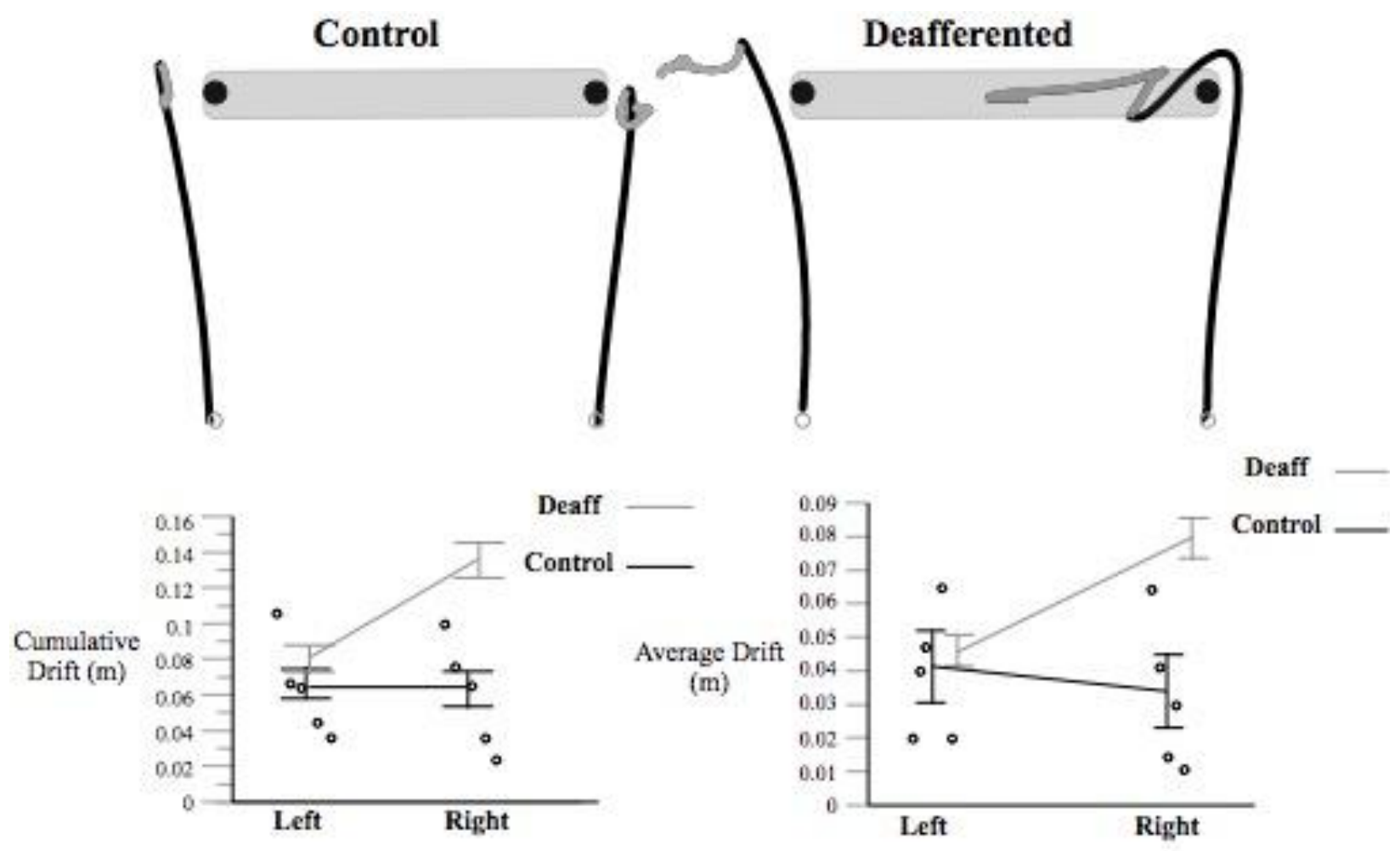

Figure 5. Movement trajectory and endpoint stabilization/drift. The top panel shows example hand paths (top view) with black reflecting the main movement and gray reflecting submovements after the initial stop. The bottom panel shows mean cumulative drift and average drift. Error bars reflect standard error across all participants for the Control group and across trials for the Deafferented patient. Black open circles represent means of individual control participants.

The characteristics of the movement that occurred after the initial stop differed between the deafferented participant and the control group. In the control group, late movement was typically corrective to move the hands closer to the target, whereas the late movement for the deafferented participant was typically associated with a drift further away from the target. Figure 6 shows our measure of the direction of drift for each hand of the control group and for the deafferented participant, which was calculated by taking the distance of each end of the bar to its target location at the end of the trial and subtracting the distance from the target at initial movement stop. If this late movement moved the bar closer to the target, that corresponded to 
negative drift, and if it moved farther away from the target, the drift was positive. As shown in Figure 6, on average, the hands of the deafferented participant drifted away from the targets, while the control group corrected to move slightly closer to the target. Case-controls comparison showed significant differences between the deafferented patient and controls, for both arms. The paired t-tests on control data revealed no significant differences between arms $(\mathrm{p}=.2583)$.

Statistical analysis using the RSDT showed that the asymmetry in the deafferented participant was significantly greater than that of the control group $(\mathrm{p}=.004)$.

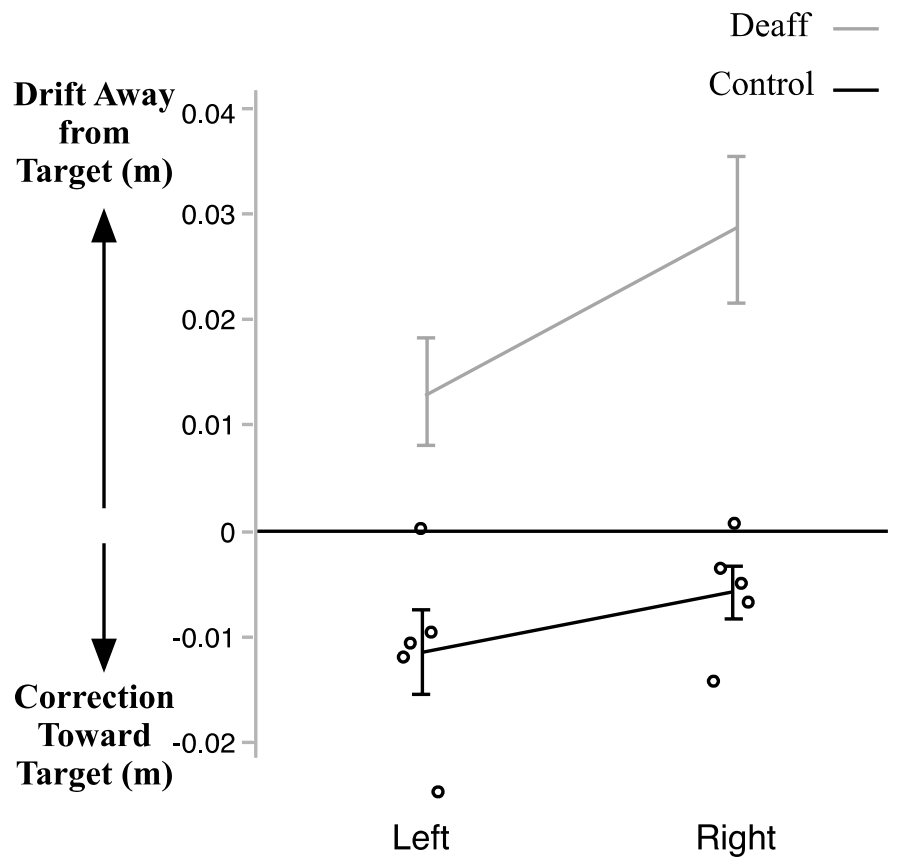

Figure 6: Drift in the proprioceptively-deafferented patient, and correction in the Control group during the secondary submovements. The graph shows whether the direction of the movement after the initial stop was toward the target (correction) or away from the target (drift). Error bars reflect standard error across all participants for the Control group and across trials for the Deafferented patient. Black open circles represent means of individual control participants.

In order to calculate an index of bimanual coordination, we examined the movement of the participants along the redundant axis of the bar. To prevent task errors, movements along 
this bar axis must be negatively correlated between the limbs such that a displacement in one direction for one arm must be matched by synchronous and matched displacement in the opposite direction for the other arm. Figure 7 shows this correlation between the left and right hands at peak velocity and at movement end for each participant along with the orthogonal fit regression line and ellipses representing the 2-D variance of each participant. At peak velocity, the deafferented participant showed less linear correlation between the hands compared with those of the control participants. At movement end, this difference was extreme, with the deafferented participant's correlation approaching zero $\left(\mathrm{R}^{2}=0.01\right)$ with a largely circular distribution, . In contrast, control participants had higher correlations with slopes approaching -1 . We quantified 2-D standard deviation, which corresponds to the area of each ellipse shown on Figure 7. Case-controls comparison showed that the deafferented participant had significantly greater 2-D standard deviation at peak velocity $(\mathrm{p}=.016)$ and at movement end $(\mathrm{p}=.002)$. This shows that interlimb coordination was significantly impaired for the deafferented participant, and that this discoordination increased during the course of movement. 


\section{Hand Correlation at Peak Velocity}

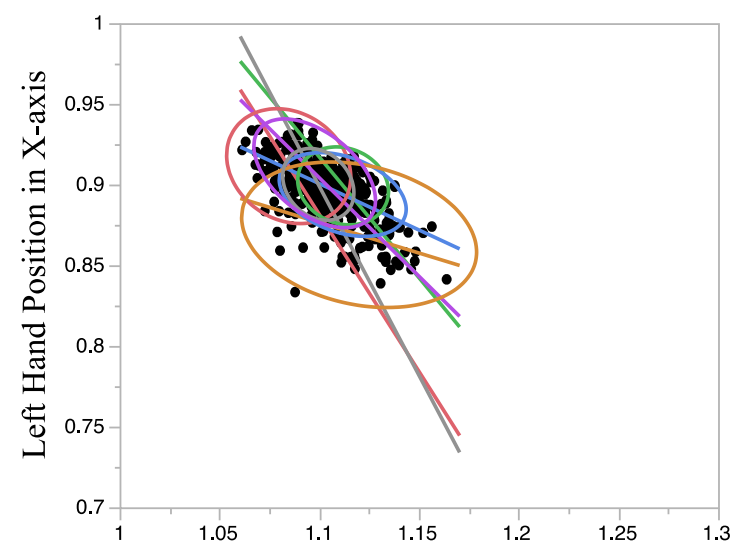

Right Hand Position in X-axis

\begin{tabular}{|l|l|l|l|}
\hline \multicolumn{1}{|c|}{ Subject } & $\begin{array}{c}\text { Slope of } \\
\text { Orthogonal Fit }\end{array}$ & \multicolumn{1}{|c|}{$\mathrm{R}^{2}$} & 2-D Std Dev \\
\hline C1 & -1.95 & -0.189 & 0.0156 \\
\hline C2 & -1.47 & -0.146 & 0.0109 \\
\hline C3 & -0.574 & -0.351 & 0.0135 \\
\hline C4 & -2.34 & -0.232 & 0.0094 \\
\hline C5 & -1.22 & -0.499 & 0.0150 \\
\hline DP & -0.378 & -0.239 & 0.0245 \\
\hline
\end{tabular}

\section{Hand Correlation at End}

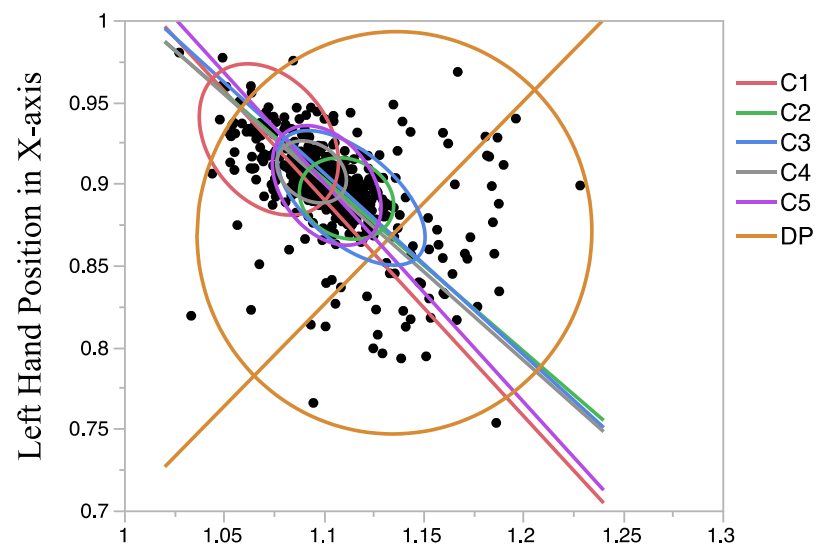

Right Hand Position in X-axis

\begin{tabular}{|l|l|l|l|}
\hline \multicolumn{1}{|c|}{ Subject } & \multicolumn{1}{|c|}{$\begin{array}{c}\text { Slope of } \\
\text { Orthogonal Fit }\end{array}$} & \multicolumn{1}{|c|}{$\mathrm{R}^{2}$} & 2-D Std Dev \\
\hline C1 & -1.33 & -0.303 & 0.0237 \\
\hline C2 & -1.05 & -0.160 & 0.0140 \\
\hline C3 & -1.11 & -0.586 & 0.0226 \\
\hline C4 & -1.09 & -0.266 & 0.0105 \\
\hline C5 & -1.34 & -0.367 & 0.0186 \\
\hline DP & 1.24 & 0.010 & 0.0645 \\
\hline
\end{tabular}

Figure 7: Correlation of left and right hand positions on bar $\mathbf{x}$-axis- Position of the right hand vs. left hand along the redundant $\mathrm{x}$-axis of the bar is plotted for each trial at peak velocity (left) and movement end (right). Each point represents one trial. Orthogonal fit regression lines are also shown for each participant along with ellipses representing the 2-D standard deviation of the data points. C1-5 denotes the control participants while DP denotes the deafferented participant. $\mathrm{R}^{2}$ and slopes of the regression line and 2-D standard deviation are quantified and shown in the tables below the graphs. 


\section{Discussion}

In this study, we examined how proprioception contributes to bimanual coordination by comparing performance in a shared bimanual task of a person with large fiber sensory neuropathy with a typical age-matched control group. We found performance deficits of the deafferented patient, with significant asymmetry in control of hand trajectory in the deafferented participant, whose movements were poorly coordinated between the two limbs. In addition, we found significant asymmetries in the drift of each hand after initial cessation of movement for the deafferented participant, such that her dominant right hand drifted away from the target more than the non-dominant left hand. In contrast, no such drift occurred for the control participants, who tended to either remain stable, or make small corrections toward the target.

\section{The role of proprioception for trajectory control of the dominant and non-dominant arms}

Both hands of the deafferented participant showed significant deficits in trajectory control, which is consistent with previous studies on unimanual movements of deafferented patients (Messier et al., 2003; Sainburg et al., 1993; Sainburg et al., 1995; Lefumat et al., 2016; Jayasinghe et al., 2020, 2021; for review, Sarlegna \& Sainburg, 2009). However, our findings also reveal that during this bimanual task, the non-dominant arm of the deafferented participant showed significantly larger trajectory deficits than the dominant arm. This asymmetry is consistent with findings from studies on healthy participants in which the dominant hand exhibits significantly straighter, and efficient movements (Sainburg, 2002).

We have previously hypothesized that this difference reflects a dominant hemisphere specialization for predictive control of intersegmental coordination, whereas the non-dominant hemisphere is more reliant on impedance control through largely feedback mechanisms 
(Sainburg, 2014; Jayasinghe et al., 2020). The fact that deafferentation has previously been shown to interfere with predictive control of intersegmental dynamics (Messier et al., 2003; Sainburg et al., 1993, 1995) is consistent with the idea that predictive control relies on an accurate model of limb dynamics that is updated by proprioceptive feedback. Our findings indicate that asymmetry in predictive and impedance control during reaching movements persists following deafferentation, supporting a feedforward role in these processes.

The finding that asymmetry in trajectory control persists in this shared bimanual task, in which symmetry between the limbs at the beginning and end of motion is required, supports and extends previous work. It has been widely reported that during simultaneous bimanual tasks, the two arms tend to perform similarly to each other whether the movements of each arm are instructed to be the same or different (Kelso, Southard, \& Goodman, 1979a; Swinnen, 2002). In symmetrical bimanual movements, healthy participants tend to show very tight coordination, particularly in temporal parameters, between the limbs. For studies in which two different movements are to be performed simultaneously by each arm, there is a tendency for the two movements to become similar, a phenomenon referred to as bimanual interference. This has led some to suggest that during bimanual movements, a shared control signal might "couple" the movements of the arms (Kelso et al., 1979a; Marteniuk, MacKenzie, \& Baba, 1984; Teasdale et al., 1994). However, asymmetries in bimanual movements have been documented (Byblow, Chua, \& Goodman, 1995; Dounskaia et al., 2010), and the lack of "coupling" found in the present study for the deafferented participant, both for trajectory and final position control, suggests that proprioceptive signals from the two arms might be critical for such spatiotemporal coupling of the two arms. Impaired correlation between hands, , impaired final position accuracy and increased bar angle during movement also provide more evidence of an impairment in 
interlimb coordination resulting from deafferentation. This provides support for the findings of Kazennikov and Wiesendanger (2005), who found that synchronization of the two hands during a bimanual reach and grab task was disrupted by altering proprioception via tendon vibration applied to one of the arms. The current results extend these findings by showing that complete loss of proprioception disrupts coordination between the arms.

The role of proprioception for postural control of the dominant and non-dominant arms

A novel finding of this study is the dramatic asymmetry in final position drift demonstrated by the deafferented participant. Large postural drifts, when required to hold the limb still without visual monitoring, has consistently been reported for deafferented individuals (Nougier et al., 1996; Rothwell et al., 1982; Sanes et al., 1985; Sarlegna et al., 2006; Jayasinghe et al., 2020, 2021). In addition, large drifts at the end of planar reaching movements have been reported (Gordon et al., 1995; Ghez et al., 1999). However, those studies examined only dominant arm movement, and thus, asymmetry in drift was not reported. The findings of impaired final position stability of the dominant arm and impaired trajectory control of the nondominant arm for the deafferented participant show that even in the absence of proprioceptive feedback, lateralized mechanisms persist, resulting in asymmetries in steady state position control and trajectory control.

Some authors have hypothesized independent neural mechanisms for controlling trajectory and stable final positions during reaching (Kurtzer, Herter, \& Scott, 2005; Scheidt \& Ghez, 2007). These mechanisms may be lateralized, as suggested by Yadav and Sainburg (Yadav \& Sainburg, 2011; 2014), who introduced a serial hybrid model to explain lateralization of simple reaching movements, in which movements are initiated with optimal trajectory control 
and terminated using a final position, or impedance, control. Consistent with the dynamic dominance hypothesis, the optimal controller had defined costs of energy, speed, and task error. Similar hybrid control models were proposed by Scheidt and Ghez (2007) and Jayasinghe et al (2020). Yadav and Sainburg (2014) showed that when the switch point between these controllers was optimally fit to dominant and non-dominant arm trajectories, the switch to impedance control occurred early in movement for the non-dominant trajectories and late in movement for the dominant arm trajectories. These findings suggested an asymmetry in the contributions of each controller to each arm, such that the optimal trajectory controller contributed greater to dominant arm movements, while the impedance controller contributed greater to non-dominant arm movements. It should be stressed that none of these simulations incorporated explicit feedback loops, but instead were based on open-loop control mechanisms. Thus, these findings predicted that asymmetries in control of trajectory and final position should persist in deafferented individuals performing movements without vision, and thus without the opportunity for feedback-based adjustments to their movements. Our current findings of impaired final position stability with the dominant arm and impaired trajectory with the non-dominant arm of a deafferented participant show that even without proprioceptive feedback, control remains lateralized such that the dominant controller is specialized for trajectory control and the nondominant is specialized for impedance control. These findings, thus, emphasize the role of feedforward processes in both of these hypothesized control processes. In addition, previous research has proposed that the mechanism of switching between trajectory and postural controllers relies on proprioceptive signals arising during the course of movement (Cordo, Carlton, Bevan, Carlton, \& Kerr, 1994; Scheidt \& Ghez, 2007; Jayasinghe et al, 2020). Thus, in the absence of proprioception, this switch might be disrupted or may not occur. This might 
explain the extreme asymmetries in postural stability between the hands in the deafferented participant. Without proprioception, dominant arm movements may never switch to the postural controller, thus leading to continuous drift after movement. In contrast, non-dominant arm movements of deafferented participants may rely totally on impedance control, accounting for the greater trajectory deviations, but more stable final positions, with lower drift.

To conclude, our current results indicate that deafferentation had differential effects on each aspect of control in the two limbs. The non-dominant left hand of the deafferented participant showed substantial deficits in trajectory coordination, but was better able to stabilize position at the end of motion, whereas, the dominant right hand showed better trajectory control, but demonstrated greater drift at the end of movement. These findings support the idea of a lateralization of the neural mechanisms underlying trajectory control and postural control in human. 


\section{Funding}

This work was supported by the National Institutes of Health Grant \# R01HD059783 to R.L.S. and a National Center for Scientific Research-France International Programmes for Scientific Cooperation (PICS) grant (to F.R.S.). 


\section{References}

Blouin, J., Bard, C., Teasdale, N., Paillard, J., Fleury, M., Forget, R., \& Lamarre, Y. (1993). Reference systems for coding spatial information in normal subjects and a deafferented patient. Experimental Brain Research, 93(2), 324-331. https://doi.org/10.1007/BF00228401

Boyd,I.A.(1965).Differences in the diameter and conduction velocity of motor and fusimotor fibres in nerves to different muscles in the hind limb of the cat. In Studies in Physiology, edited by D. R. Curtis, and A. K. McIntyre, pp. 7-12. Springer, Berlin, and Heidelberg.

Bozzacchi, C., Cimmino, R. L., \& di Russo, F. (2017). The temporal coupling effect: Preparation and execution of bimanual reaching movements. Biological Psychology, 123, 302-309. https://doi.org/10.1016/j.biopsycho.2016.10.016

Byblow, W. D., Chua, R., \& Goodman, D. (1995). Asymmetries in Coupling Dynamics of Perception and Action. Journal of Motor Behavior, 27(2), 123-137. https://doi.org/10.1080/00222895.1995.9941705

Cooke, J. D., Brown, S., Forget, R., \& Lamarre, Y. (1985). Initial agonist burst duration changes with movement amplitude in a deafferented patient. Experimental Brain Research, 60(1), 184-187. https://doi.org/10.1007/BF00237030

Cordo, P., Carlton, L., Bevan, L., Carlton, M., \& Kerr, G. K. (1994). Proprioceptive coordination of movement sequences: role of velocity and position information. Journal of Neurophysiology, 71(5), 1848-1861. https://doi.org/10.1152/jn.1994.71.5.1848

Crawford, J. R., \& Garthwaite, P. H. (2005). Testing for suspected impairments and dissociations in single-case studies in neuropsychology: Evaluation of alternatives using Monte Carlo simulations and revised tests for dissociations. Neuropsychology, 19(3), 318-331. https://doi.org/10.1037/0894-4105.19.3.318

Crawford, J. R., Garthwaite, P. H., \& Porter, S. (2010). Point and interval estimates of effect sizes for the case-controls design in neuropsychology: Rationale, methods, implementations, and proposed reporting standards. Cognitive Neuropsychology, 27(3), 245-260. https://doi.org/10.1080/02643294.2010.513967

Cuadra, C., Falaki, A., Sainburg, R., Sarlegna, F. R., \& Latash, M. L. (2019). Case Studies in Neuroscience: The central and somatosensory contributions to finger interdependence and coordination: lessons from a study of a "deafferented person". Journal of Neurophysiology, 121(6), 2083-2087. https://doi.org/10.1152/jn.00153.2019

Diedrichsen, J. (2007). Optimal Task-Dependent Changes of Bimanual Feedback Control and Adaptation. Current Biology, 17(19), 1675-1679. https://doi.org/10.1016/j.cub.2007.08.051

Diedrichsen, J, \& Dowling, N. (2009). Bimanual coordination as task-dependent linear control policies. Human Movement Science, 28(3), 334-347.

https://doi.org/10.1016/j.humov.2008.10.003

Dounskaia, N., Nogueira, K. G., Swinnen, S. P., \& Drummond, E. (2010). Limitations on Coupling of Bimanual Movements Caused by Arm Dominance: When the Muscle Homology Principle Fails. Journal of Neurophysiology, 103(4), 2027-2038. https://doi.org/10.1152/jn.00778.2009

Drewing, K., Stenneken, P., Cole, J., Prinz, W., \& Aschersleben, G. (2004). Timing of bimanual movements and deafferentation: implications for the role of sensory movement effects. Experimental Brain Research, 158(1), 50-57. https://doi.org/10.1007/s00221-004-1870-9

Forget, R., \& Lamarre, Y. (1987). Rapid elbow flexion in the absence of proprioceptive and cutaneous feedback. Human Neurobiology, 6(1), 27-37. 
Ghez C, Krakauer J, Sainburg RL, Ghilardi MF (1999) Spatial representations and internal models of limb dynamics in motor learning. In: Gazzaniga MS (ed) The New Cognitive Neurosciences. The MIT Press, Cambridge, MA, pp. 501-514.

Ghez, C., \& Sainburg, R. (1995). Proprioceptive control of interjoint coordination. Canadian Journal of Physiology and Pharmacology, 73, 273-284. https://doi.org/10.1139/y95-038

Gordon, J., Ghilardi, M. F., Cooper, S. E., \& Ghez, C. (1994). Accuracy of planar reaching movements. II. Systematic extent errors resulting from inertial anisotropy. Experimental Brain Research, 99(1), 112-130. https://doi.org/10.1007/BF00241416

Gordon, J., Ghilardi, M. F., \& Ghez, C. (1995). Impairments of Reaching Movements in Patients Without Proprioception . I . Spatial Errors. Journal of Neurophysiology, 73(1).

Helmuth, L. L., \& Ivry, R. B. (1996). When two hands are better than one: reduced timing variability during bimanual movements. Journal of Experimental Psychology. Human Perception and Performance, 22(2), 278-293.

Jayasinghe, S. A. L., Sarlegna, F. R., Scheidt, R. A., \& Sainburg, R. L. (2020). The neural foundations of handedness: insights from a rare case of deafferentation. Journal of Neurophysiology, 124(1), 259-267. https://doi.org/10.1152/jn.00150.2020

Jayasinghe, S. A. L., Sarlegna, F. R., Scheidt, R. A., \& Sainburg, R. L. (in press). Somatosensory deafferentation reveals lateralized roles of proprioception in feedback and adaptive feedforward control of movement and posture. Current Opinion in Physiology. https://doi.org/10.1016/j.cophys.2020.10.005

Kazennikov, O. V, \& Wiesendanger, M. (2005). Goal synchronization of bimanual skills depends on proprioception. Neuroscience Letters, 388(3), 153-156. https://doi.org/10.1016/j.neulet.2005.06.040

Kelso, J. A., Southard, D. L., \& Goodman, D. (1979a). On the coordination of two-handed movements. Journal of Experimental Psychology. Human Perception and Performance, 5(2), 229-238.

Kelso, J. A., Southard, D. L., \& Goodman, D. (1979b). On the nature of human interlimb coordination. Science (New York, N.Y.), 203(4384), 1029-1031. https://doi.org/10.1126/science.424729

Kurtzer, I., Herter, T. M., \& Scott, S. H. (2005). Random change in cortical load representation suggests distinct control of posture and movement. Nature Neuroscience, 8(4), 498-504. https://doi.org/10.1038/nn1420

Lafargue, G., Paillard, J., Lamarre, Y., \& Sirigu, A. (2003). Production and perception of grip force without proprioception: is there a sense of effort in deafferented subjects? The European Journal of Neuroscience, 17(12), 2741-2749.

Lefumat, H. Z., Miall, R. C., Cole, J. D., Bringoux, L., Bourdin, C., Vercher, J. L., \& Sarlegna, F. R. (2016). Generalization of force-field adaptation in proprioceptively-deafferented subjects. Neuroscience Letters, 616, 160-165. https://doi.org/10.1016/j.neulet.2016.01.040

Lajoie, Y., Teasdale, N., Cole, J. D., Burnett, M., Bard, C., Fleury, M., ... Lamarre, Y. (1996). Gait of a deafferented subject without large myelinated sensory fibers below the neck. Neurology, 47(1), 109-115. https://doi.org/10.1212/wnl.47.1.109

Marteniuk, R. G., MacKenzie, C. L., \& Baba, D. M. (1984). Bimanual Movement Control: Information processing and Interaction Effects. The Quarterly Journal of Experimental Psychology Section A, 36(2), 335-365. https://doi.org/10.1080/14640748408402163

Messier, J., Adamovich, S., Berkinblit, M., Tunik, E., \& Poizner, H. (2003). Influence of movement speed on accuracy and coordination of reaching movements to memorized 
targets in three-dimensional space in a deafferented subject. Experimental Brain Research, 150(4), 399-416.

Miall, R. C., Rosenthal, O., Ørstavik, K., Cole, J. D., \& Sarlegna, F. R. (2019). Loss of haptic feedback impairs control of hand posture: a study in chronically deafferented individuals when grasping and lifting objects. Experimental Brain Research, 237(9), 2167-2184. https://doi.org/10.1007/s00221-019-05583-2

Mutha, P. K., \& Sainburg, R. L. (2009). Shared Bimanual Tasks Elicit Bimanual Reflexes During Movement. Journal of Neurophysiology, 102(6), 3142-3155. https://doi.org/10.1152/jn.91335.2008

Nougier, V., Bard, C., Fleury, M., Teasdale, N., Cole, J., Forget, R., ... Lamarre, Y. (1996). Control of single-joint movements in deafferented patients: evidence for amplitude coding rather than position control. Experimental Brain Research, 109(3), 473-482. https://doi.org/10.1007/BF00229632

Rothwell, J. C., Traub, M. M., Day, B. L., Obeso, J. A., Thomas, P. K., \& Marsden, C. D. (1982). Manual motor performance in a deafferented man. Brain : A Journal of Neurology, 105 (Pt 3), 515-542. https://doi.org/10.1093/brain/105.3.515

Sainburg, R. L. (2002). Evidence for a dynamic-dominance hypothesis of handedness. Experimental Brain Research, 142(2), 241-258. https://doi.org/10.1007/s00221-001-0913-8

Sainburg, Robert L. (2014). Convergent models of handedness and brain lateralization. Frontiers in Psychology, 5(SEP), 1-14. https://doi.org/10.3389/fpsyg.2014.01092

Sainburg, Robert L, \& Ghez, C. (1993). Loss of Proprioception Produces Deficits in Interjoint Coordination. Journal of Neurophysiology, 70(5), 2136-2147.

Sainburg, Robert L, Ghilardi, M. F., Poizner, H., \& Ghez, C. (1995). Control of Limb Dynamics in Normal Subjects and Patients Without Proprioception. Journal of Neurophysiology, $73(2)$.

Sanes, J. N., Mauritz, K. H., Dalakas, M. C., \& Evarts, E. v. (1985). Motor control in humans with large-fiber sensory neuropathy. Human Neurobiology, 4(2), 101-114.

Sarlegna, F. R., Gauthier, G. M., Bourdin, C., Vercher, J.-L., \& Blouin, J. (2006). Internally driven control of reaching movements: a study on a proprioceptively deafferented subject. Brain Research Bulletin, 69(4), 404-415. https://doi.org/10.1016/j.brainresbull.2006.02.005

Sarlegna, F. R., Malfait, N., Bringoux, L., Bourdin, C., \& Vercher, J. L. (2010). Force-field adaptation without proprioception: Can vision be used to model limb dynamics? Neuropsychologia, 48(1), 60-67. https://doi.org/10.1016/j.neuropsychologia.2009.08.011

Sarlegna, F. R., \& Sainburg, R. L. (2009). The roles of vision and proprioception in the planning of reaching movements. Advances in experimental medicine and biology, 629, 317-335. https://doi.org/10.1007/978-0-387-77064-2_16

Schaffer, J. E., Maenza, C., Good, D. C., Przybyla, A., \& Sainburg, R. L. (2020). Left hemisphere damage produces deficits in predictive control of bilateral coordination. Experimental Brain Research, 238(12), 2733-2744. https://doi.org/10.1007/s00221-02005928-2

Schaffer, J. E., \& Sainburg, R. L. (2020). Interlimb Responses to Perturbations of Bilateral Movements are Asymmetric. Journal of Motor Behavior, 1-17. Advance online publication. https://doi-org.ezaccess.libraries.psu.edu/10.1080/00222895.2020.1760196

Scheidt, R. A., \& Ghez, C. (2007). Separate Adaptive Mechanisms for Controlling Trajectory and Final Position in Reaching. Journal of Neurophysiology, 98(6), 3600-3613. https://doi.org/10.1152/jn.00121.2007 
Spencer, R. M. C., Ivry, R. B., Cattaert, D., \& Semjen, A. (2005). Bimanual coordination during rhythmic movements in the absence of somatosensory feedback. Journal of Neurophysiology, 94(4), 2901-2910. https://doi.org/10.1152/jn.00363.2005

Swinnen, S. P. (2002). Intermanual coordination: from behavioural principles to neural-network interactions. Nature Reviews. Neuroscience, 3(5), 348-359. https://doi.org/10.1038/nrn807

Swinnen, S. P., Dounskaia, N., \& Duysens, J. (2002). Patterns of bimanual interference reveal movement encoding within a radial egocentric reference frame. Journal of Cognitive Neuroscience, 14(3), 463-471. https://doi.org/10.1162/089892902317361976Teasdale N, Bard C, Fleury M, Paillard J, Forget R, Lamarre Y (1994) Bimanual interference in a deafferented patient and control subjects. In: Swinnen SP, Heuer H, Massion J, Casaer P. Interlimb coordination : neural, dynamical and cognitive constraints. pp 243-258. Orlando: Academic Press.

Yadav, V., \& Sainburg, R. L. (2011). Motor lateralization is characterized by a serial hybrid control scheme. Neuroscience, 196, 153-167.

Yadav, V., \& Sainburg, R. L. (2014). Limb dominance results from asymmetries in predictive and impedance control mechanisms. PLOS ONE, 9(4).

https://doi.org/10.1371/journal.pone.0093892 